\title{
Clinically relevant behavioral endpoints in a recurrent nitroglycerin migraine model in rats
}

Kenneth J. Sufka ${ }^{1,2,3^{*}}$, Stephanie M. Staszko ${ }^{1}$, Ainslee P. Johnson ${ }^{1}$, Morgan E. Davis ${ }^{1}$, Rachel E. Davis ${ }^{1}$ and Todd A. Smitherman ${ }^{1}$

\begin{abstract}
Background: This research sought to further validate the rat nitroglycerin (NTG) migraine model by comparing the effects of single versus recurrent NTG episodes on behavioral endpoints that mirror ICHD-3 diagnostic criteria for migraine, and to determine if the altered behavioral endpoints are reduced after administration of sumatriptan.

Methods: Separate cohorts of rats were administered NTG $(10 \mathrm{mg} / \mathrm{kg} / 2 \mathrm{ml}$ ) or saline (Experiment 1: single injection; Experiment 2: repeated injections; Experiment 3: repeated injections with sumatriptan [0.0, 0.3 and $1.0 \mathrm{mg} / \mathrm{kg} / \mathrm{ml}]$ rescue. Behavioral endpoints were assessed $2 \mathrm{~h}$ after final NTG administration and included time in light/dark chambers for photophobia and activity, pain facial ratings, and cool $\left(5^{\circ} \mathrm{C}\right)$ and warm $\left(46^{\circ} \mathrm{C}\right)$ tail dip.

Results: The first two experiments demonstrated that repeated $(n=5)$ but not single NTG injections produced photophobia, decreased activity, and yielded less weight gain than saline injections. Experiment 3 showed that sumatriptan attenuated hypoactivity, reduced facial expressions of pain, and reversed weight alterations in a dosedependent manner.
\end{abstract}

Conclusions: These findings identify numerous clinical homologies of a recurrent NTG rat migraine model that may be useful for screening novel pharmacotherapies.

Keywords: Migraine, Nitroglycerin, Headache, Translational research, Animal models

\section{Background}

Migraine affects $12-16 \%$ of population annually $[1,2]$ and is the third most common medical condition in the world [3], contributing to significant disability and reduced quality of life [3, 4]. Despite its prevalence and impact, migraine remains undertreated [1] and its pathophysiology incompletely understood. In part these challenges stem from a need for more translational research using wellestablished animal simulations [5], in which homologies are demonstrated between the human clinical presentation and animals' behavioral responses. Although rodents have been used in pain research for well over six decades, work to validate a migraine simulation is a comparatively recent

\footnotetext{
* Correspondence: pysufka@olemiss.edu

1Department of Psychology, University of Mississippi, Oxford, MS 38677, USA

${ }^{2}$ Research Institute of the Pharmaceutical Sciences, University of Mississippi,

Oxford, MS 38677, USA

Full list of author information is available at the end of the article
}

undertaking. Although no single model is likely to explain all aspects of migraine [6], animal simulations have already informed our understanding of the genetic basis of familial hemiplegic migraine and the role of cortical spreading depression in aura $[7,8]$.

Existing animal models of migraine vary considerably in their methods of inducing migraine. Two common approaches include peripheral administration of nitroglycerin (NTG) [9] or intracranial administration of inflammatory mediators $[10,11]$. NTG administration produces attacks phenotypically similar to spontaneous migraine attacks $[8,12]$ and sensitizes trigeminal and cortical structures that underlie migraine pain [13]. Most rodent NTG models use a single injection, but in humans migraine presents as disorder involving recurrent episodic manifestations [14]. One recent study has shown that a model using repeated NTG injections 
holds promise for studying the ontogeny of migraine as a chronic disorder [15].

Rodent migraine models often use endpoints that include thermal or tactile allodynia as indicators of migraine-related sensory hypersensitivity $[7,10,16]$. Allodynia is reported by nearly $2 / 3$ of migraineurs [17] but is conceptualized primarily as a marker of migraine progression [18, 19], infrequently assessed clinically [20], and not a diagnostic criterion [14]. Thus, existing rodent models may not adequately map onto the human clinical presentation as they rarely quantify spontaneously emitted pain behaviors $[21,22]$, which comprise patients' primary reason for seeking treatment.

The aims of the present research thus were to further validate the NTG migraine model in rats by comparing the effects of single versus multiple NTG episodes on behavioral endpoints that reflect ICHD-3 diagnostic symptoms of migraine, including pain intensity, photophobia, and alterations in motor activity. Changes in body weight and thermal allodynia were also assessed. An additional validation step was to determine if NTG-sensitive endpoints were reduced after administration of sumatriptan.

\section{Methods}

\section{Ethics, consent, and permissions}

Housing and experimental procedures were approved by the university's IACUC (protocol \#13-023).

\section{Subjects and housing characteristics}

Separate cohorts of naïve male Sprague-Dawley rats (Harlan Laboratories; Indianapolis, IN, USA) weighing 250-330 g served as subjects for each of three experiments; animals were randomized to treatment condition. Rats were pair-housed in $13 \times 21 \times 22 \mathrm{~cm}$ polyethylene tubs. Food (Teklad 7001, Teklad Diets, Madison, WI, USA) and water were available ad libitum. Room temperature was maintained at $22+/-4{ }^{\circ} \mathrm{C}$, and overhead florescent illumination was maintained on a 12/12-h cycle (lights on at 07:00). Testing procedures were carried out during the middle third of the light cycle.

Experiments 2 and 3 involved repeated NTG-migraine episodes over a 2-week period with testing following the last episode. Small igloo-type enclosures (PVC end caps $16.5 \mathrm{~cm} \times 7.5 \mathrm{~cm}$ with painted black interior and arched opening cut into the sidewall) were placed in the home cages to permit rats to reduce exposure to colony room lighting after NTG injections.

\section{Drugs}

Nitroglycerin (NTG; SDM ${ }^{\circ} 27$, Copperhead Chemical, Tamaqua, PA, USA) was diluted in a 50:50 propylene glycol and ethanol vehicle. NTG dose was $10 \mathrm{mg} / \mathrm{kg}$ based on previous studies that this dose reliably produced alterations in allodynia $2 \mathrm{~h}$ after administration $[15,16]$. To avoid the possibility of migraine-related activity of ethanol in the vehicle, saline was administered to the non-migraine control group. NTG or saline was administered in blinded fashion intraperitoneally (IP) in a volume of $2 \mathrm{ml} / \mathrm{kg}$. For Experiment 3, sumatriptan succinate (PAR Pharmaceutical, Woodcliff Lake, New Jersey, USA) was dissolved in physiological saline to doses of 0.3 and $1.0 \mathrm{mg} / \mathrm{kg} / \mathrm{ml}$ and delivered IP.

\section{Behavioral measures}

All behavioral measures were collected by researchers blinded to treatment condition.

\section{Rat Grimace Scale (RGS)}

RGS scores were used as an index of pain severity; scoring of facial action units is described in detail elsewhere [23]. RGS has been quantified in inflammatory, neuropathic, and incision pain models but this is the first attempt to score facial action units in a model of migraine. Photos were captured using a digital camera from rats placed in $31 \times 22 \times 26 \mathrm{~cm}$ Plexiglas chambers. Photographs were randomly ordered then scored by two trained assistants; rater scores were averaged, and percent agreement was $81 \%$ across studies.

\section{Tail flick test}

Warm- and cold-water tail flick tests were conducted to assess thermal allodynia. Though not an ICHD-3 criterion for migraine, allodynia was included to index sensory hypersensitivity and for comparison with prior animal studies. Water temperature in $250 \mathrm{ml}$ beakers was maintained at $46{ }^{\circ} \mathrm{C}+/-0.1{ }^{\circ} \mathrm{C}$ using a hot plate or at $15{ }^{\circ} \mathrm{C}+/-0.1{ }^{\circ} \mathrm{C}$ using crushed ice. For testing, each rat was gently wrapped in a terry cloth towel and its tail submerged $5 \mathrm{~cm}$. Latency to flick or curl the tail was recorded with a 40 -s cut-off.

\section{Light/dark box}

A place preference apparatus (Model\# MED-CPP-013, Med Associates, St. Albans, VT, USA) was modified to create a light/dark box used to quantify the ICHD-3 cr iteria of photophobia and reduced activity associated with migraine. Clear lids of the black and center gray chambers were covered with heavy black construction paper (inside $\leq 5$ lux); the white chamber with clear lids served as the light portion (inside $\geq 635 \mathrm{lux}$ ). On test day, rats were placed into the center chamber for a $1 \mathrm{~m}$ acclimation period after which the guillotine-style doors were opened to allow access to the entire apparatus. Time in the light chamber and total number of photobeam breaks during a $10 \mathrm{~m}$ test session were recorded via vendor software. 
Elevated plus maze (EPM) and forced swim test (FST)

Because frequent (ie "chronic") migraineurs show increased rates of anxiety and depression [24-26], experiment 2 added EPM and FST assays to determine if multiple injections induced these symptoms. The EPM is a widely used assay to model generalized anxiety disorder and was used to quantify anxiety-like behavior that may be associated with repeated migraine inductions in Experiment 2. The apparatus, positioned $76 \mathrm{~cm}$ above the floor, consisted of four arms $(56 \mathrm{~cm})$, two of which have $19 \mathrm{~cm}$ tall walls creating the "closed" arms, while "open" arms resemble an open runway. For testing, rats were placed onto the maze center facing an open arm. Distressed rodents display increased avoidance of the open arms. During a $5 \mathrm{~m}$ test session, time spent in the open arms was recorded via EthoVision video tracking software (Noldus, Leesburg VA, USA).

The FST is a commonly used model of behavioral despair used to quantify depression-like behavior and was used in Exp 2 for that purpose. Rats were placed into a $20 \mathrm{~cm}$ diameter $\times 35 \mathrm{~cm}$ tall cylinder filled with $24-26{ }^{\circ} \mathrm{C}$ water to within $5 \mathrm{~cm}$ of the rim. Rats initially attempt to escape through swimming and diving; distressed rats display decreased latency to behavioral despair (ie, floating behavior). Time engaged in floating behavior, which included small movements necessary for the rat to keep its head above water, during a $5 \mathrm{~m}$ test session served as the dependent measure and was quantified by EthoVision video tracking software.

\section{Statistical analyses}

Data were analyzed using SPSS with independent samples t-tests, one-way (between groups) ANOVAs, or two-way (between and within groups) ANOVAs with post-hoc tests for simple effects (Fisher's exact or independent samples t-tests); significance was $p<.05$.

\section{EXPERIMENT 1: single NTG vs. Saline administration}

\section{Procedure}

Rats received either $10 \mathrm{mg} / \mathrm{kg}$ NTG or saline $(n=9-10$ per group) IP and then were returned to their home cages. Photographs for RGS scoring were taken 60, 90, and $120 \mathrm{~m}$ post-injections with rats returned to their home cages between sessions. After the last photograph, rats were tested in adjacent lab on the tail flick tests (counter-balanced for order), followed by light/dark box for photophobia and locomotion.

\section{Results}

As summarized in Table 1, NTG significantly altered only the behavioral endpoint of locomotor activity. In contrast
Table 1 Effects of a single NTG migraine episode on behavioral endpoints

\begin{tabular}{lll}
\hline Endpoints & Saline & NTG \\
\hline Grimace Score (60-90-120 m) & $.057 / .025 / .063$ & $.163 / .207 / .188$ \\
& $(.096 / .053 / .106)$ & $(.289 / .379 / .319)$ \\
Cool-Tailflick Latency (s) & $16.8(10.0)$ & $19.2(13.6)$ \\
Warm-Tailflick Latency (s) & $8.9(6.2)$ & $12.3(5.7)$ \\
Time in Light Box (s) & $27.2(12.7)$ & $40.4(19.6)$ \\
Locomotor Activity (\# beam breaks) & $105.8(29.0)$ & $217.8^{*}(79.4)$
\end{tabular}

Means (SD)

${ }^{*} p<.05$ vs saline control

to our prediction the NTG group exhibited more activity than the saline group, $\mathrm{t}(15)=3.957, p=.022$.

\section{Discussion}

A single NTG episode produced negligent effects on photophobia, RGS, and allodynia. Increased locomotor activity of NTG rats may reflect escape-like behavior associated with ongoing pain. Noting that ICHD-3 diagnosis of migraine requires a history of multiple episodes, Experiment 2 sought to determine whether repeated NTG injections induce alterations in these behavioral endpoints and whether repeated injections induced symptoms of anxiety or depression.

\section{EXPERIMENT 2: repeated NTG episodes}

\section{Procedure}

Three groups of rats ( $n=10$ per group) received 5 IP injections over 2 weeks (every third day during the middle of their light cycle). Control rats received 5 saline administrations; experimental groups received either 3-NTG (alternated with 2 saline) or 5-NTG administrations. NTG doses were the same as in Experiment 1. Behavioral measures described earlier were conducted $2 \mathrm{~h}$ after the fifth injection in the aforementioned order. Rats were tested on the EPM the following morning and the FST that afternoon.

\section{Results}

As shown in Table 2, a main effect for body weight growth was found, $\mathrm{F}(2,27)=6.93, p=.004$. Planned comparisons confirmed that 3- and 5-NTG groups gained significantly less weight than controls ( $p s=.005$ and .021 ). As before, repeated NTG did not produce significant alterations in RGS or tail-flick latencies nor affect time in the EPM or FST. As shown in Fig. 1, however, repeated administrations produced a main effect for time spent in the light portion of the light-dark box (Panel A), $F(2,27)=5.97$, $p=.007$. Planned comparisons revealed the 5-NTG group spent less time in the light chamber than the 
Table 2 Effects of recurrent NTG migraine episodes on behavioral endpoints

\begin{tabular}{llll}
\hline Endpoints & $\begin{array}{l}\text { 0-NTG } \\
\text { (5 Saline) }\end{array}$ & $\begin{array}{l}\text { 3-NTG } \\
\text { (2 Saline) }\end{array}$ & 5-NTG \\
\hline \% Change Body Weight & $21.76(3.21)$ & $14.93^{*}(7.49)$ & $13.42^{*}(4.35)$ \\
Grimace Score & $.075(.105)$ & $.138(.181)$ & $.20(.206)$ \\
Cool-Tailflick Latency (s) & $16.5(12.4)$ & $23.0(18.3)$ & $20.1(15.6)$ \\
Warm-Tailflick Latency (s) & $9.8(5.3)$ & $10.4(3.4)$ & $13.2(12.5)$ \\
EPM-Time in Open Arms (s) & $13.0(19.3)$ & $17.4(16.7)$ & $16.2(22.9)$ \\
FST-Float Time (s) & $136.3(36.1)$ & $144.5(41.4)$ & $112.9(46.0)$ \\
\hline
\end{tabular}

Mean (SD); EPM elevated plus maze. FST forced swim test ${ }^{*} p<.05$ vs 0 -NTG control

0 -NTG group $(p=.005)$; a similar pattern was seen in the 3-NTG group $(p=.055)$. Repeated NTG episodes also yielded a main effect for locomotor activity (Panel B), $\mathrm{F}(2,27)=4.53, p=.02$, in which mean photobeam breaks in the 3- and 5-NTG groups were significantly lower than the $0-\mathrm{NTG}$ control group ( $p \mathrm{~s}=.036$ and .04 , respectively).

\section{Discussion}

Repeated NTG episodes produced significant alterations in weight gain, light sensitivity and locomotor activity. Reduction in weight gain may be associated with reduced appetite that often accompanies migraine but this requires future exploration. In contrast to the aforementioned migraine-specific effects, repeated NTG administration did not produce thermal allodynia nor affect stress-related behaviors on the EPM and FST, the latter of which suggests that increased frequency of episodes may be necessary to model affective comorbidities that accompany chronic migraine. While NTG-induced thermal allodynia has been detected using the Hargreaves procedure [9], our findings suggest that this does not present to the tail.

Taken in conjunction with Experiment 1, repeated NTG administration accompanied by measures of clinicallyrelevant behavioral endpoints of light sensitivity and reduced locomotor activity better simulate features of human migraineurs and begin to establish the model's validity. An additional validation step was to determine whether these endpoints are affected by sumatriptan, a common migraine abortive.

\section{EXPERIMENT 3: sumatriptan effects on repeated NTG episodes}

\section{Procedure}

Three groups of rats ( $n=8-10$ per group) received a series of 5 NTG injections over 2 weeks (every third day during the middle third of their light cycle). Thirty minutes after each administration, rats received either saline or 0.3 or $1.0 \mathrm{mg} / \mathrm{kg}$ sumatriptan IP and were returned to their home cages. RGS and light/dark box assays were conducted $90 \mathrm{~m}$ after the fifth administration. FST and EPM assays were not included in Experiment 3 because evidence across behavioral assays in Experiment 2 confirmed the utility of the 5-NTG proto$\mathrm{col}$ in modeling episodic but not chronic migraine nor its associated affective comorbidities.

\section{Results}

Though sumatriptan had modest effects on body weight change, a significant main effect was found for RGS, $\mathrm{F}(2,24)=6.23, p=.007$, such that the mean RGS scores for both sumatriptan groups were significantly lower than the saline group ( $p s<.006)$, confirming that sumatriptan reduced pain intensity (see Table 3 ). Sumatriptan did not significantly affect photophobia as all rats spent little time in the light portion of the box (Fig. 2, Panel a) but dose-dependently attenuated reductions in locomotor activity in a manner that approached significance, $\mathrm{F}(2,24)=2.40, p=0.112$. Planned comparisons revealed that animals treated with $1.0 \mathrm{mg} / \mathrm{kg}$ sumatriptan had significantly more photobeam breaks (increased activity) than the saline group $(p<0.045)$.

\section{Discussion}

Results demonstrate that at least one of the clinicallyrelevant endpoints of repeated NTG-migraine can be attenuated by sumatriptan (i.e., hypoactivity). The inability to reverse alterations in light sensitivity may be related to testing in a novel environment after only the final NTG episode, and exposure to the box after each injection might afford detection of more robust drug effects. Interestingly, sumatriptan attenuated RGS scores and alterations in body weight associated with repeated NTG episodes. Differences in body weight changes between Experiments 2 and 3 likely reflect differing initial body weights due to limits in animal availability from the vendor: Experiment 2 used younger rats within their main growth curve function while Exp 3 used older rats nearer their body weight plateau. Whether body weight changes reflect an indirect measure of nausea or reduced appetite remains to be determined. We found quantification of RGS to be challenging as the observed alterations in facial action units are not as robust as seen in other pain models (mean RGS scores >1.0 are found in neuropathic and arthritic models), and thus believe RGS is unlikely to be a suitable behavioral endpoint for modeling migraine.

\section{Conclusions}

The overall aim of these three studies was to further validate an NTG migraine model in rodents by employing an endophenotypic mapping strategy that asked two 

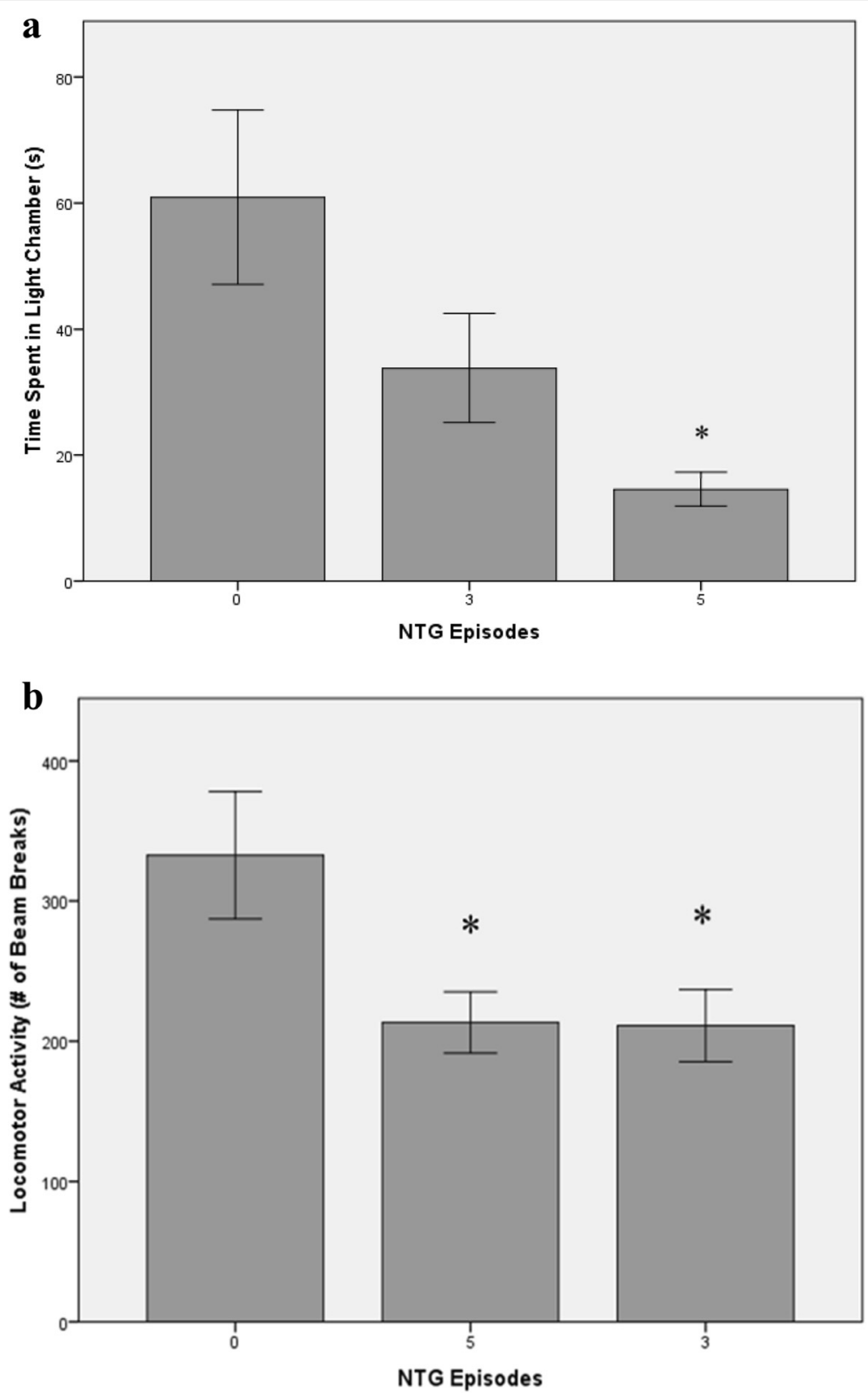

Fig. 1 Behavioral endpoints of light sensitivity (a) and motor activity (b) as a function of NTG injection condition $(M \pm S E M){ }^{*} p<.05$ vs. saline control

Table 3 Effects of sumatriptan on body weight and grimace scores after five NTG episodes

\begin{tabular}{llll}
\hline Endpoints & Saline & $\begin{array}{l}\text { Sumatriptan } \\
0.3 \mathrm{mg} / \mathrm{kg}\end{array}$ & $\begin{array}{l}\text { Sumatriptan } \\
1.0 \mathrm{mg} / \mathrm{kg}\end{array}$ \\
\hline \% Change Body Weight & $-3.03(4.11)$ & $-0.06(4.81)$ & $1.84^{*}(4.33)$ \\
Grimace Score & $.55(.50)$ & $.14^{\mathrm{a}}(.14)$ & $.09^{*}(.11)$ \\
\hline
\end{tabular}

Mean (SD)

${ }^{*} p<.05$ vs saline control questions related to establishing homologies in symptoms and pharmacological response: First, do single or multiple NTG injections produce behavioral endpoints that simulate the clinical presentation of spontaneouslyemitted migraine symptoms in humans? Second, does sumatriptan attenuate these endpoints in rats as it does in humans? Multiple injections were used to mirror the minimum headache attacks necessary for diagnosis [14] and behavioral endpoints chosen that mapped onto ICHD-3 diagnostic criteria (e.g., photophobia, aggravation by activity).

Our results indicate that recurrent NTG episodes are necessary to affect clinically-relevant symptoms of light 

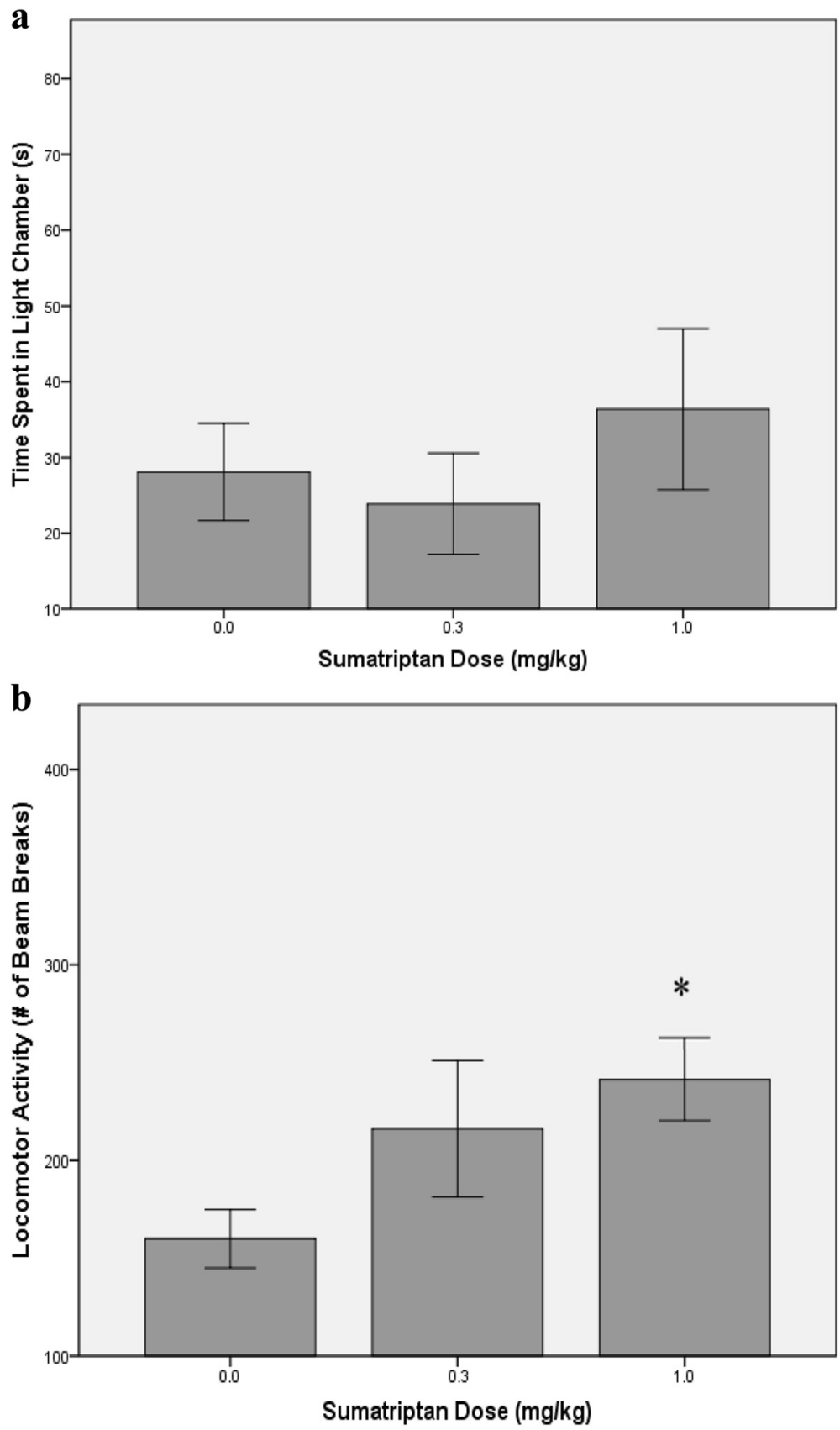

Fig. 2 Behavioral endpoints of light sensitivity (a) and motor activity (b) as a function of sumatriptan condition $(M \pm S E M){ }^{*} p<.05$ vs. saline control

sensitivity and activity in a rat migraine model. Other laboratories have reported that multiple NTG episodes may model processes of migraine progression [15], and our findings add behavioral endpoints to strengthen this clinical simulation. Finally, sumatriptan administration successfully modulated activity but not photophobia in the light-dark box; this may be attributable to an absence of exposure to the Fight-dark box during prior NTG episodes preventing avoidance learning.
Current research in our lab is exploring protocol variations that may increase effects on the clinically-relevant behavioral endpoints. Once accomplished, several lines of research may further validate this recurrent NTG migraine model. For example, this model should be sensitive to established migraine preventive pharmacotherapies [15], as well as novel abortive treatments such as those targeting cannabinoid receptors [27]. Utilization of more frequent NTG injections over longer time periods may 
better model the phenomenon of chronic migraine and associated states of allodynia and comorbid psychiatric symptomatology $[18,26]$. Another promising line of research would be to determine whether these endpoints are influenced by estrous cycle stage or manifest in other models of migraine induction $[10,11]$. Collectively, the work described herein and that of other researchers in modeling migraine will ultimately provide better simulations with greater translational value, leading to further insights into both the pathophysiology and treatment of this common and debilitating condition.

\section{Competing interests}

The authors declare they have no competing interests.

\section{Authors' contributions}

KUS and TAS conceived of the study. RED participated in the design of the study. SMS, APJ, and MED collected data. KJS, RED, and TAS drafted the manuscript. SMS, APJ, and MED helped revise the manuscript for intellectual content. All authors approved the final version and agree to be accountable for the work.

\section{Author details}

'Department of Psychology, University of Mississippi, Oxford, MS 38677, USA. ${ }^{2}$ Research Institute of the Pharmaceutical Sciences, University of Mississippi, Oxford, MS 38677, USA. ${ }^{3}$ Department of Pharmacology, University of Mississippi, Oxford, MS 38677, USA.

Received: 27 January 2016 Accepted: 7 April 2016

Published online: 19 April 2016

\section{References}

1. Lipton RB, Bigal ME, Diamond M et al (2007) Migraine prevalence, disease burden, and the need for preventive therapy. Neurology 68:343-349

2. Smitherman TA, Burch $\mathrm{R}$, Sheikh $\mathrm{H}$ et al (2013) The prevalence, impact and treatment of migraine and severe headaches in the United States: A review of statistics from national surveillance studies. Headache 53:427-436

3. Vos T, Flaxman AD, Naghavi M et al (2012) Years lived with disability (YLDs) for 1160 sequelae of 289 diseases and injuries 1990-2010: A systematic analysis for the Global Burden of Disease Study 2010. Lancet 380:2163-2196

4. Leonardi M, Steiner TJ, Scher AT et al (2005) The global burden of migraine: measuring disability in headache disorders with WHO's Classification of Functioning, Disability, and Health (ICF). J Headache Pain 6:429-440

5. Olesen J, Jansen-Olesen I (2012) Towards a reliable animal model of migraine. Cephalalgia 32:578-580

6. Bergerot A, Holland PR, Akerman S et al (2006) Animal models of migraine: looking at the component parts of a complex disorder. Eur J Neurosci 24:1517-1534

7. Andreou AP, Summ O, Charbit AR et al (2010) Animal models of headache: From bedside to bench and back to bedside. Expert Rev Neurother 10:389-411

8. Buzzi MG, Tassorelli C (2010) Experimental models of migraine. In: Nappi G, Moskowitz MA (eds) Handbook of Clinical Neurology (Vol 97: Headache). Elsevier, New York, pp 109-123

9. Bates EA, Nikai T, Brennan KC et al (2010) Sumatriptan alleviates nitroglycerin-induced mechanical and thermal allodynia in mice. Cephalagia 30:170-178

10. Edelmayer RM, Le LN, Xan J et al (2012) Activation of TRPA1 on dural afferents: A potential mechanism of headache pain. Pain 153:1949-1958

11. Yan J, Melemedjian OK, Price TJ et al (2012) Sensitization of dural afferents underlies migraine-related behavior following meningeal application of interleukin-6 (IL-6). Mol Pain 8:6. doi:10.1186/1744-8069-8-6

12. Thomsen LL, Kruuse C, Iversen HK et al (1994) A nitric oxide donor (nitroglycerin) triggers genuine migraine attacks. Eur J Neurol 1:73-80

13. Di Clemente L, Coppola G, Magis D et al (2009) Nitroglycerin sensitizes in healthy subjects CNS structures involved in migraine pathophysiology: Evidence from a study of nociceptive blink reflexes and visual evoked potentials. Pain 144:156-161
14. Headache Classification Committee of the International Headache Society (2013) The International Classification of Headache Disorders, $3^{\text {rd }}$ ed. Cephalalgia 33:629-808

15. Pradhan AA, Smith ML, McGuire B et al (2014) Characterization of a novel model of chronic migraine. Pain 155:269-274

16. Costa A, Smeraldi A, Tassorelli C et al (2005) Effects of acute and chronic restraint stress on nitroglycerin-induced hyperalgesia in rats. Neurosci Lett 383:7-11

17. Lipton RB, Bigal ME, Ashina A et al (2008) Cutaneous allodynia in the migrain epopulation. Ann Neurol 63:148-158

18. Louter MA, Bosket JE, van Oosterhout WPJ et al (2013) Cutaneous allodynia as a predictor of migraine chronification. Brain 136:3489-3496

19. Burstein R, Collins B, Jakubowski M (2004) Defeating migraine pain with triptans: a raceagainst the development of cutaneous allodynia. Ann Neurol 55:19-26

20. Mathew NT, Kailasam J, Seifert T (2004) Clinical recognition of allodynia in migraine. Neurology 63:848-852

21. Mogil JS (2009) Animal models of pain: progress and challenges. Nat Rev Neurosci 10:283-294

22. Mogil JS, Crager SE (2004) What should we be measuring in behavioral studies of chronicpain in animals? Pain 112:12-15

23. Sotocinal S, Sorge R, Zaloum A et al (2011) The Rat Grimace Scale: A partially automated method for quantifying pain in the laboratory rat via facial expressions. Mol Pain 7:55

24. Saunders K, Merikangas K, Low NCP et al (2008) Impact of comorbidity on headache-related disability. Neurology 70:538-547

25. Kim SK, Park SP (2014) Suicidal ideation and risk factors in Korean migraine patients. J Clin Neurosci 21:1699-1704

26. Buse DC, Manack A, Serrano D et al (2010) Sociodemographic and comorbidity profiles of chronic migraine and episodic migraine sufferers. J Neurol Neurosurg Psychiatry 81:428-432

27. Greco R, Mangione AS, Sandrini G et al (2014) Activation of CB2 receptors as a potential therapeutic target for migraine: Evaluation in an animal model. J Headache Pain 15:14. doi:10.1186/1129-2377-15-14

\section{Submit your manuscript to a SpringerOpen ${ }^{\circ}$ journal and benefit from:}

- Convenient online submission

- Rigorous peer review

- Immediate publication on acceptance

- Open access: articles freely available online

- High visibility within the field

Retaining the copyright to your article

Submit your next manuscript at $>$ springeropen.com 